\title{
The challenge and the reproach of infective endocarditis
}

Sir,

We have the following comments to make on Professor Goodwin's editorial (Br Heart $\mathcal{F}$ 1985; 54: 115-8).

(a) We know of no evidence that fungal endocarditis has increased, and certainly not as the result of "intensive and lengthy therapy for advanced bacterial infective endocarditis." We therefore do not agree with the recommendation for weekly prophylactic amphotericin. Nor do we know of any evidence that the immunocompromised are especially vulnerable to endocarditis.

(b) It is not known whether appropriate prophylaxis will prevent endocarditis although it is assumed that many potential cases are prevented by it.

(c) The recommendation of the British Society for Antimicrobial Chemotherapy Working Party is for a single oral dose of $\mathbf{3 g}$ not $\mathbf{2 g}$ of amoxycillin for dental prophylaxis.

(d) Not all "streptococci likely to have come from the mouth" can be satisfactorily killed in vitro with penicillin alone. Thus treatment with penicillin alone is inappropriate, as indeed is ampicillin alone for faecal streptococci.

(e) Potassium penicillin in large intravenous doses can cause hyperkalaemia, but it is no longer available for parenteral use.

(f) The recommendations for gentamicin blood concentrations in streptococcal endocarditis might result in toxicity. The working party recommended that gentamicin should be given in a low dose to enchance the activity of penicillin. Peak blood concentrations of $3-5 \mathrm{mg} / 1$ and trough blood concentrations $<1 \mathrm{mg} / 1$ are sufficient for this purpose. Gentamicin should seldom be given for more than two weeks. Gentamicin can be used in combination with flucloxacillin to treat staphylococcal endocarditis and here the dose will need to be greater than that for streptococcal infection to achieve peak blood concentrations of $5-10 \mathrm{mg} / 1$ and trough blood concentrations of $<2 \mathrm{mg} / \mathrm{l}$.

(g) Early prosthetic endocarditis with Staphylococcus aureus is nearly always the result of sternal wound infection and resultant bacteraemia. The aetiology of late prosthetic endocarditis is quite different from early onset infection. It is caused by the same spectrum of organisms as those causing natural valve infection.

(h) Antibiotic resistance of the infecting organism is seldom the reason for a fatal outcome in endocarditis. The mortality of this disease remains high because of late diagnosis, poor choice and incorrect dosage of antibiotics, and, more important, late referral to cardiologists and cardiac surgeons.

(i) The most important initial investigations in suspected endocarditis are blood cultures. An erythrocyte sedimentation rate and haemoglobin are not a substitute because they are non-specific. Where "viridans streptococci" are cultured from the blood the diagnosis is nearly always endocarditis and sometimes it may come as a surprise.

Susannah Eykyn

on behalf of the working party on Endocarditis of the British Society for Antimicrobial Chemotherapy

Department of Microbiology,

St Thomas' Hospital,

London SE1 7EH.

This letter was shown to Professor Goodwin, who replies as follows:

Sir,

I welcome the opportunity of replying to Dr Eykyn's important comments as her letter gives me the chance to reinforce the message in my editorial that early diagnosis and early consultation with the cardiac surgeons are important for success in treatment and reduction of mortality.

(a) I know of no evidence that fungal infection has increased nor did I quote any. I suggested that "fungal infection may paradoxically have increased as a result of more intensive and lengthy chemotherapy for advanced bacterial infective endocarditis." This is a personal view based on personal experience, and I believe this view may be shared by others. ${ }^{1}$

I pointed out that "patients who are immunologically compromised, such as those taking immunosuppressive drugs are especially vulnerable to infection of any sort." I do not think that any experienced clinician or microbiologist would seriously challenge this statement. I did not quote any 
evidence that such patients were especially vulnerable to infective endocarditis, though it seems reasonable to suppose that they might be. ${ }^{2}$ Immunosuppressed patients are listed among those specially vulnerable to infective endocarditis in the latest report of the Royal College of Physicians of London Research Unit. ${ }^{3}$

(b) I agree that it is assumed that many potential cases of infective endocarditis are prevented by appropriate prophylaxis, and I pointed out that "Although prophylaxis remains a somewhat imprecise exercise, it should at least be meticulously applied to patients who are especially at risk."

(c) The dose of $2 \mathrm{~g}$ of amoxycillin by mouth one hour before dental work for outpatients was an error for which I make due apology. The correct dose as recommended by the working party of the British Society for Antimicrobial Chemotherapy ${ }^{4}$ is indeed a single dose of $3 \mathrm{~g}$. It is of interest though, that Shanson et al in $1978^{5}$ showed that a single dose of $2 \mathrm{~g}$ amoxycillin given one hour before tooth extraction reduced the incidence of bacteraemia detected from 14 of 14 controls to two out of 14 given amoxycillin, and a single $2 \mathrm{~g}$ dose was advised by Oakley in $1979 .{ }^{6}$ Two years later, Shanson et al reported that $3 \mathrm{~g}$ of amoxycillin effectively prevented bacteraemia after dental extraction. ${ }^{7}$

(d) I entirely agree that penicillin alone is not appropriate for treatment of streptococcal or staphylococcal endocarditis, as I wrote under the heading of Treatment: "Since over $80 \%$ of infections are due to staphylococci or streptococci, initial treatment should be with a combination of drugs, such as gentamicin and penicillin." I did not suggest that ampicillin alone should be given for faecal streptococcal infections.

(e) I am grateful to Dr Eykyn for emphasising that potassium penicillin is not available for parenteral use.

(f) The gentamicin blood levels that I advocated, based on personal experience and published recommendations, ${ }^{8}$ are indeed somewhat different from those quoted by Dr Eykyn - that is peak blood concentrations of 3-5 mg/l and trough concentrations of $<1 \mathrm{mg} / \mathrm{l}$. I said that peak concentrations should not exceed $14 \mathrm{mg} / 1$ or fall below $5 \mathrm{mg} / 1$ and trough concentrations should not exceed $2 \mathrm{mg} / \mathrm{l}$. The Guide to Antimicrobial Therapy published by the Use of Drugs Subcommittee of the Ealing, Hounslow and North Hammersmith District in 1981 states that the gentamicin nomogram of Mawer et al ${ }^{9}$ is designed to give serum concentrations of gentamicin within the range $3-10 \mathrm{mg} / 1$ two hours after each intramuscular dose.

(g) I do not agree that early prosthetic staphylococcal endocarditis is "nearly always" the result of sternal wound infection. Certainly the sternal wound may be the source of the infection, but entry of staphylococci from the patient's skin when the drains are removed is important. (h) I wrote that "reasons for the failure (to reduce mortality) are multiple and include organisms resistant to antibiotics"... I agree that this is not the most frequent reason for failure and I mentioned other causes including more direct exposure of the heart to infection by open operation, insertion of foreign bodies into the heart, and older and sicker patients who develop the disease. I agree entirely that the high mortality of infective endocarditis is due mainly to delay in diagnosis, and late referral to cardiologists and cardiac surgeons as I stated clearly in the editorial. The use of incorrect doses of antibiotics is a less common though not an unimportant reason.

(i) Of course the most important investigation is blood culture. But a high erythrocyte sedimentation rate though it is not specific can be a very useful warning to investigate further in a suggestive clinical situation. The test is simpler and gives an earlier result than blood culture. I agree that streptococci of the viridans group in the blood nearly always indicate endocarditis, but the diagnosis rarely comes "as a surprise" to experienced cardiologists and clinicians.

I make no apology for quoting personal views and experience, because I feel that it is the function of an editorial to express these and provoke lively discussion.

\section{J F Goodwin,}

Emeritus Professor of Clinical Cardiology,

Royal Postgraduate Medical School,

London W120HS.

\section{References}

1 Gray IR. Management of infective endocarditis. $\mathcal{F} R$ Coll Physicians Lond 1981; 15: 173-8.

2 Bayliss R, Clarke C, Oakley CM, Somerville W, Whitfield AGW, Young SEJ. The microbiology and pathogenesis of infective endocarditis. Br Heart $\mathcal{~} 1$ 1983; 50: 513-9.

3 Bayliss R, Clarke C, Oakley CM, Somerville W, Whitfield AGW, Young SEJ. Incidence, mortality and prevention of infective endocarditis. $\mathcal{f} R$ Coll Physicians Lond 1986; 20: 15-20.

4 British Society for Antimicrobial Therapy. The antibiotic prophylaxis of infective endocarditis. Report of a working party. Lancet 1984; ii: 1323-6.

5 Shanson DC, Cannon P, Wilks M. Amoxycillin compared with penicillin $\mathrm{V}$ for the prophylaxis of dental bacteraemia. I Antimicrob Chemother 1978; 4: 431-6.

6 Oakley CM. Prevention of infective endocarditis. Tho$\operatorname{rax} 1979$; 34: 711-2.

7 Shanson DC, Ashford RFU, Singh J. High-dose oral amoxycillin for preventing endocarditis. $\mathrm{Br} \mathrm{Med} \mathrm{f}$ 1980; 280: 446-8.

8 Oakley CM. Infective endocarditis. $\mathrm{Br} f$ Hosp Med 1980; 24: 232-7.

9 Mawer GE, Ahmed R, Dobbs SA, McGough JG, Lucas SB, Tooth JA. Prescribing aids for gentamycin. Br f Clin Pharmacol 1974; 1: 45-50. 